\title{
Detecting Single-Nucleotides by Tunneling Current Measurements at Sub-MHz Temporal Resolution
}

\author{
Takanori Morikawa, Kazumichi Yokota, Sachie Tanimoto, Makusu Tsutsui * \\ and Masateru Taniguchi
}

The Institute of Scientific and Industrial Research, Osaka University, 81 Mihogaoka, Ibaraki 567-0047, Japan; takanori.morikawa32@sanken.osaka-u.ac.jp (T.M.); yokota@sanken.osaka-u.ac.jp (K.Y.); sachie.tanimoto32@sanken.osaka-u.ac.jp (S.T.); taniguti@sanken.osaka-u.ac.jp (M.T.)

* Correspondence: tsutsui@sanken.osaka-u.ac.jp; Tel.: +81-6-6879-8447

Academic Editor: W. Rudolf Seitz

Received: 22 January 2017; Accepted: 14 April 2017; Published: 18 April 2017

\begin{abstract}
Label-free detection of single-nucleotides was performed by fast tunneling current measurements in a polar solvent at $1 \mathrm{MHz}$ sampling rate using $\mathrm{SiO}_{2}$-protected $\mathrm{Au}$ nanoprobes. Short current spikes were observed, suggestive of trapping/detrapping of individual nucleotides between the nanoelectrodes. The fall and rise features of the electrical signatures indicated signal retardation by capacitance effects with a time constant of about 10 microseconds. The high temporal resolution revealed current fluctuations, reflecting the molecular conformation degrees of freedom in the electrode gap. The method presented in this work may enable direct characterizations of dynamic changes in single-molecule conformations in an electrode gap in liquid.
\end{abstract}

Keywords: tunneling current; single molecule; sequencing

\section{Introduction}

Tunneling current measurements in liquid have been considered a promising way to identify the sequence of base molecules in RNA and DNA [1-3]. The method uses a pair of metal electrodes, mostly made of $\mathrm{Au}$, with nanometer separation to detect temporal changes in the two-probe $\mathrm{dc}$ current at several to $10 \mathrm{kHz}$ sampling rates upon trapping/detrapping of individual nucleobases in the nanogap [4-8]. Single-molecule signals were then obtained in forms of current spikes, whose height represents the electron transport through a molecule. Although the signal height and width vary widely due to the sensitive nature of the tunneling conductance on the molecular conformations, it was demonstrated that the types of nucleotides can be discriminated by analyzing and comparing the statistical distributions of the current spike height [4-10].

Tremendous efforts have been undertaken to realize applications of the tunneling current approach for practical uses by incorporating nanopore technologies to the quantum mechanical approach, which enables the active drawing of single-polynucleotides in the gap by means of an electrophoretic control of the translocation dynamics [11-15]. In contrast to the progress, however, little efforts have been made to evaluate and enhance the temporal resolution of the tunneling current measurements, which is an important issue in respect to the nanopore sequencing wherein the constituent nucleobase molecules in DNA move through the electrode gap swiftly within microseconds [12,13]. In this study, therefore, we developed a nanoelectrode system for single-nucleotide detections by tunneling current measurements at $1 \mathrm{MHz}$ sampling rates in liquid (Figure 1). We used insulator-protected mechanically-controllable break junctions (MCBJs) [16-18] to form nanotip-exposed Au electrodes of $1 \mathrm{~nm}$ separation. The feasibility of the nanoprobes was evaluated by carrying out single-nucleotide detections in a polar solvent at room temperature on the basis of fast tunneling current measurements at $1 \mathrm{MHz}$. 


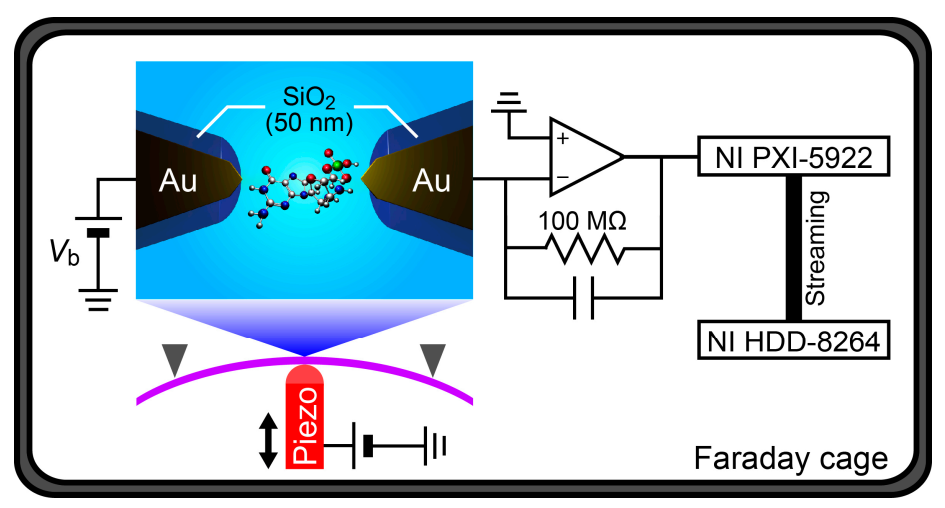

Figure 1. Schematic description of the tunneling current measurement setup. $\mathrm{SiO}_{2}$-coated $\mathrm{Au}$ nanoelectrodes were connected to feedthrough for biasing the dc voltage $V_{\mathrm{b}}$ and amplifying the current by the amplifier. The output voltage was digitized and stored in a hard disk drive.

\section{Materials and Methods}

$\mathrm{SiO}_{2}$-coated MCBJs were fabricated by the following processes (Figure 2). First, a polyimide layer was formed on a phosphor bronze substrate by spin coating an imide precursor (Aldrich Co., London, UK), followed by baking for polymerization. On the polymer surface, a microelectrode pattern was delineated by a photolithography method using AZ-5206E resist (Zeon Co., Tokyo, Japan). After development, a 40-nm thick Au layer with a 5-nm thick $\mathrm{Cr}$ adhesion layer was deposited by radio-frequency magnetron sputtering. The sample substrate was then immersed in N,N-dimethylformaide (Wako Co., Tokyo, Japan) overnight and ultrasonicated for lift-off. Further, nanojunctions were drawn by an electron-beam lithography using ZEP520A resist (Zeon Co.). Subsequently, a 100-nm thick Au layer together with a 1-nm thick $\mathrm{Cr}$ adhesion layer was deposited by the sputtering process. As a result, we obtained Au nanojunctions after lift-off in $\mathrm{N}, \mathrm{N}$-dimethylformamide. Finally, we dry-etched the polyimide layer by a reactive ion etching with oxygen etchant gas to partially free the junctions from the substrate. Here, the length of the free-standing Au nanobridges was designed to be approximately $2 \mu \mathrm{m}$, which is an optimal design for the MCBJ setup used in the present study; while shorter bridges would render better mechanical stability and finer control of the electrode gaps, they also become critically difficult to break by bending the substrate. The actual displacement rate was calibrated by analyzing the interelectrode distance dependence of the tunneling current, as described elsewhere [16,18].

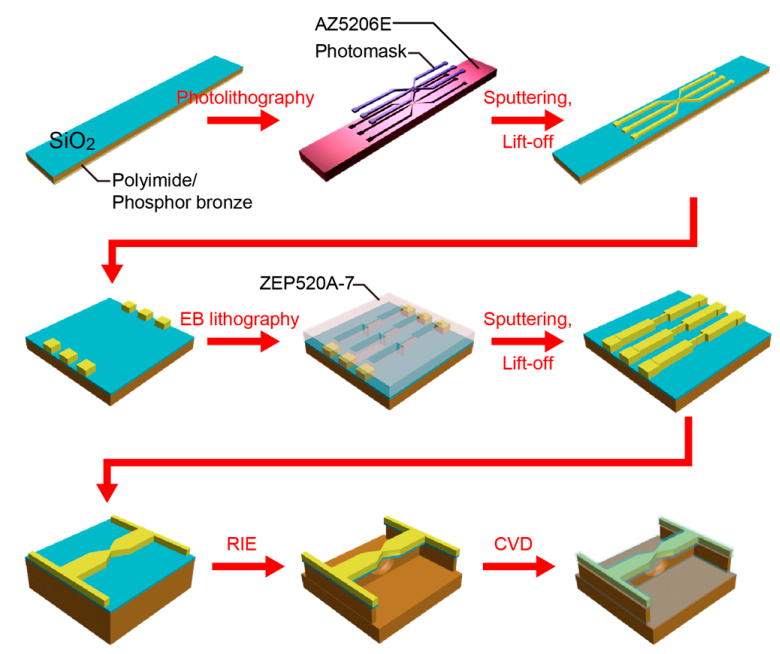

Figure 2. Fabrication processes of $\mathrm{SiO}_{2}$-coated mechanically-controllable break junctions (MCBJs). 
In the experiments, an MCBJ sample was mounted on a stage in a three-point bending configuration. Then, a dilute solution $(0.1 \mathrm{mM})$ of deoxycytidine monophosphate (dCMP) or deoxyguanosine monophosphate (dGMP) was poured into a Teflon cell attached to the MCBJ. The phosphor bronze substrate was then deflected by bending it using a piezo-driven pushing rod. Meanwhile, the conductance $G$ of the junction was measured under the applied dc bias voltage $V_{\mathrm{b}}$ using a picoammeter/source unit (Keithley 6487). By bending the substrate, $G$ tended to decrease gradually due to necking deformation at the narrowest constriction of the junction via tensile force. Further bending led the junction to break, as denoted by the $G$ drop to zero. The thus-created electrode gap was adjusted to be $1 \mathrm{~nm}$ by the piezo-control (details are described in [18]).

After forming the nanoelectrodes, the measurement unit was switched from the picoammeter to a custom-built current amplifier based on an operational amplifier ADA4817 and a $100 \mathrm{M} \Omega$ feedback resistance with a gain and bandwidth of $10^{8} \mathrm{~V} / \mathrm{A}$ and $>1 \mathrm{MHz}$ [19], respectively. The voltage source was also changed to a battery for the sake of attaining low noise. The two-probe current was then measured at $V_{\mathrm{b}}=0.5 \mathrm{~V}$ by recording the amplifier output using a digitizer (NI PXI-5922) and a RAID system (NI HDD-8264) at a $1 \mathrm{MHz}$ sampling rate. More than three MCBJ devices were used to correct the tunneling current spike data for each nucleotide measured.

\section{Results}

Fast current measurements generally involve increased noise. On the other hand, the single-nucleotide conductance only provides current changes as much as sub nanoamperes at $V_{\mathrm{b}}=0.5 \mathrm{~V}[4,5]$. We therefore tested several organic solvents, dodecane, 1,2,4-trichlorobenzene (TCB), and dimethyl sulfoxide (DMSO), in addition to water in order to obtain a low-noise condition for the single-molecule detections. The noise was characterized in terms of the power spectrum density $S_{\mathrm{N}}$ calculated from the current versus time curves recorded in the solvent at $50 \mathrm{kHz}$ sampling rates with $100 \mathrm{kHz}$ low-pass filtering (Figure 3). The noise spectra showed linear components at the frequency $f$ above $10^{2} \mathrm{~Hz}$. This feature is naturally interpreted as stemming from the voltage noise in the current amplifier coupled to the net capacitance of the MCBJ system $[19,20]$. It is noticeable that the slope is steeper in DMSO and Milli-Q compared to that in dodecane and TCB. As a result, we obtained the lowest peak-to-peak noise $I_{\mathrm{p}-\mathrm{p}}$ in dodecane and TCB (Figure 4). The noise increased by a factor of 1.7 and 2.8 in a polar organic solvent, dimethylsulphoxide (DMSO), and water, respectively. This suggests the importance of the role of the capacitance of electric double layers (EDLs) formed on the biased electrode surface that serve to increase $I_{\mathrm{p}-\mathrm{p}}$ through interaction with the noise in the amplifier voltage $[19,20]$; while the polar DMSO as well as water molecules accumulate on the electrode surface to screen the electric field there, dodecane is non-polar and hence does not form dense EDLs. More specifically, relative polarity indices $P_{\mathrm{x}}$ of the solvents are $P_{\text {water }}>P_{\mathrm{DMSO}}>P_{\text {dodecane }} \sim P_{\mathrm{TCB}}$ [21], which yields the EDL capacitance, and hence $I_{\mathrm{p}-\mathrm{p}}$, of the same order. In this respect, dodecane or TCB was found to be the best choice for the single-molecule detections. Unfortunately, however, nucleotides cannot be dissolved in the non-polar solvent. We therefore employed DMSO and Milli-Q to perform the single-molecule tunneling current measurements.
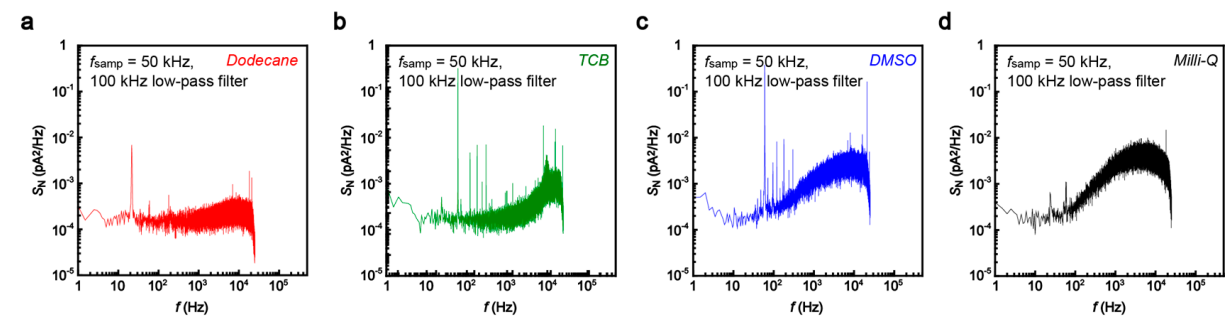

Figure 3. (a-d), Noise spectra obtained from the current versus time curves recorded at $50 \mathrm{kHz}$ with a $100 \mathrm{kHz}$ low-pass filter using an Au electrode gap of a size larger than $1 \mathrm{~nm}$ in dodecane (a); 1,2,4-trichrolobenzene (b); dimethyl sulfoxide (c); and Milli-Q (d). 


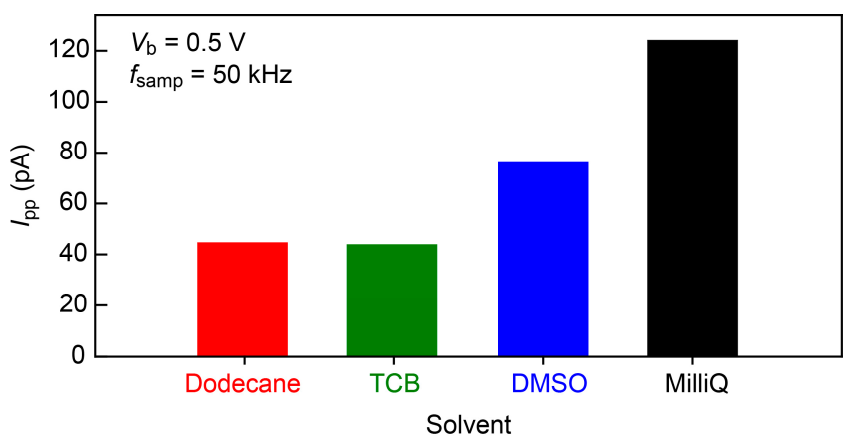

Figure 4. Solvent dependence of current noise measured with $1 \mathrm{~nm}$-sized $\mathrm{SiO}_{2}$-protected $\mathrm{Au}$ electrode gaps. Sampling rate was $50 \mathrm{kHz} .100 \mathrm{kHz}$ low pass filter was used for the current measurements. The noise is described as peak-to-peak values obtained in dimethyl sulfoxide (DMSO), dodecane, 1,2,4-trichrolobenzene (TCB), and Milli-Q.

Figure 5 displays a current $(I)$ versus time $(t)$ trace obtained in a DMSO solution of monomer dCMP at $1 \mathrm{MHz}$ sampling rate and $V_{\mathrm{b}}=0.5 \mathrm{~V}$. We observed $I$ spikes suggestive of a transient increase in the tunneling current upon single-nucleotide trapping/detrapping between an electrode gap (Figure 5a). A close look at each pulse signal revealed fast current fluctuations attributable to dynamic changes of the molecular conformations in a stochastic manner (Figure 5b, middle) [13]. It is also worth noting that the $I-t$ feature is somewhat blunt.
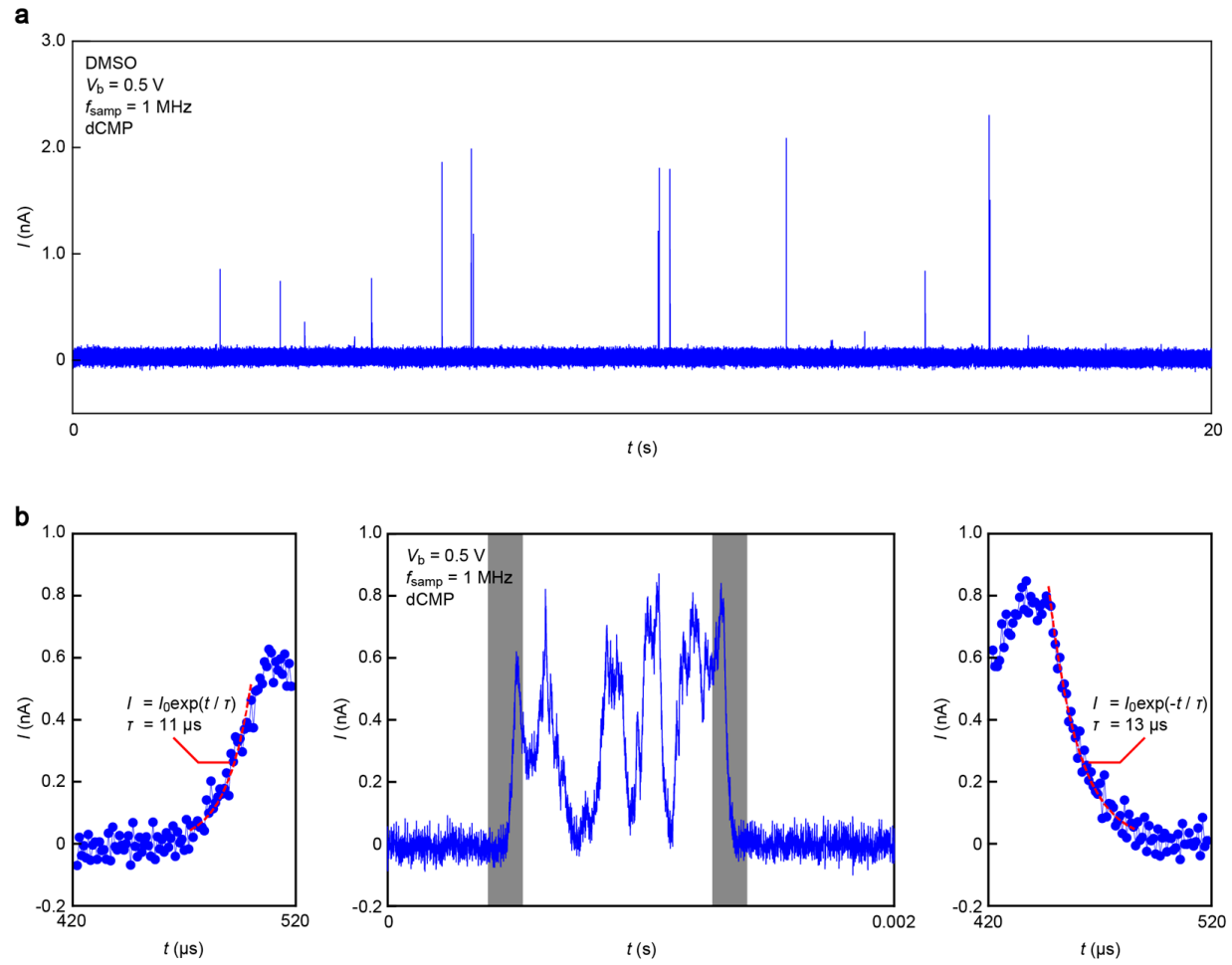

Figure 5. (a) Current versus time trace recorded in a DMSO solution of dCMP using a $1 \mathrm{~nm}$-sized $\mathrm{SiO}_{2}$-protected $\mathrm{Au}$ electrode gap at a $1 \mathrm{MHz}$ sampling rate with a $1 \mathrm{MHz}$ low-pass filter under the applied voltage $V_{\mathrm{b}}$ of $0.5 \mathrm{~V}$. Pulse-like signals were observed, signifying trapping/detrapping of single-molecule dCMP between the nanoprobes; (b) Magnified views of a tunneling current spike. Grey regions in the middle panel indicates the rising (left) and falling (right) of the signal. Red dashed lines represent the exponential fit. 
The smooth changes in I can be attributed to the slow dynamical motions of dCMPs being captured and escaping from the electrode gaps. However, the molecular motion-derived tunneling current fluctuations were predicted to take place at a pico-second time-scale [13]. The microsecond response of $I$ is, therefore, an unlikely intrinsic characteristic of tunneling transport through Au-dCMP-Au systems. On the other hand, previous studies reported significant $R C$ contributions to retard the rapid change of the tunneling current detected in aqueous media due in part to large capacitance at the EDLs formed on the current sensing electrode surface, as well as that of the insulator layer [19]. Indeed, we found exponential changes in $I$ in the rise and fall traces (Figure $5 \mathrm{~b}$, left and right panels), suggesting the influence of the capacitance effects. For these curves, numerical fitting gave the time constant $\tau$ of $11 \mu \mathrm{s}$ and $13 \mu \mathrm{s}$, respectively, during the single-molecule trapping and detrapping. Furthermore, a statistical analysis of $\tau$ calculated for 78 spikes measured showed a monomodal distribution centered at $7.5 \mu \mathrm{s}$ (Figure 6). This indicates the existence of resistance and capacitance components amounting $R C=7.5 \mu \mathrm{s}$ in the measurement circuit, including the MCBJ device used. Meanwhile, the non-Gaussian distribution at the high- $\tau$ regime would be attributed to non-specific adsorption of nucleotides on the Au surface virtually slowing down the detrap dynamics.

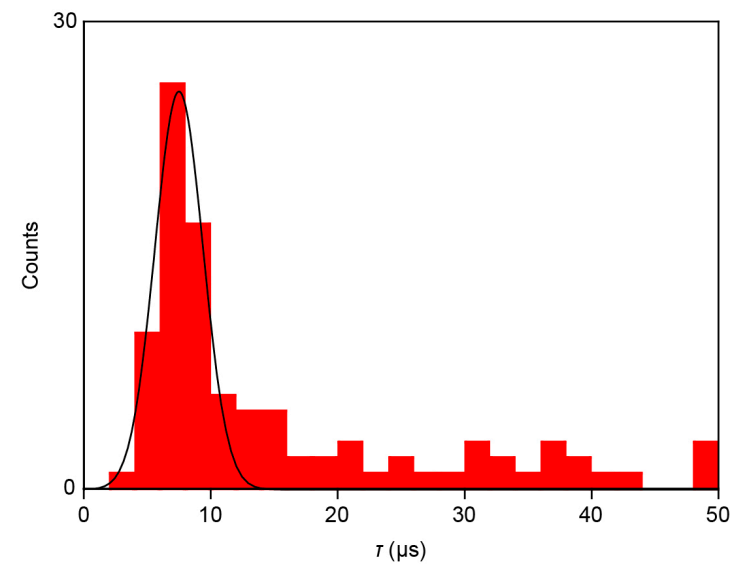

Figure 6. Statistical distribution of the time constant $\tau$. Solid curve is a Gaussian fit.

To shed further light on this point as well as to testify the feasibility of the $1 \mathrm{MHz}$ current sampling in more practical conditions, we extended the tunneling current measurements in diluted PBS buffer $(\times 0.01)$ for detections of $\mathrm{dCMP}$ and dGMP. In contrast to the noise characteristics in the air gap (Figure 7a) demonstrating mostly Johnson noise contributions, we observed pronounced capacitance effects $\left(S_{N} \sim f\right)$ in Milli-Q as well as in the buffer solution at $f>10^{2} \mathrm{~Hz}$, as expected from the high ion concentration condition that leads to formation of dense EDLs on the electrode surface (Figure 7c). Moreover, ions in the salt solution give pronounced flicker noise that further deteriorates the noise condition, giving $I_{\mathrm{pp}}$ of $447 \mathrm{pA}$ at $V_{\mathrm{b}}=0.5 \mathrm{~V}$. Fortunately, however, the noise level was not critical for detecting the tunneling current signatures of nucleotides. Figure 8 displays typical spike signals obtained for dCMP and dGMP (Figure 8a) in the diluted buffer demonstrating large fluctuations of the tunneling current attributable to dynamic changes in the molecular conformations in the electrode gap. $\tau$ deduced from the spike tails revealed monomodal distributions positioned at $\tau=6.7 \mu$ s and $6.9 \mu \mathrm{s}$ for dCMP and dGMP, respectively (Figure $8 b, c$ ), which is quite close to that in DMSO. The fact that $\tau$ changes little between the two nucleotides indicates the predominant influence of signal retardation by the $R C$ effects on $\tau$. 
a

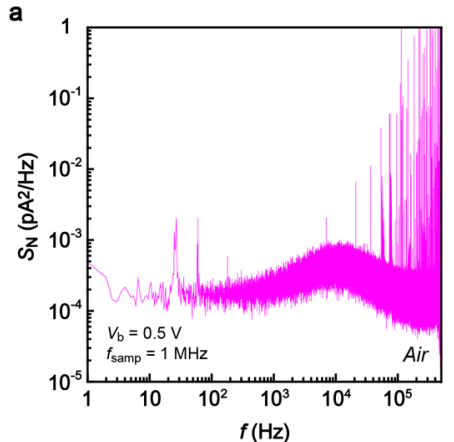

b

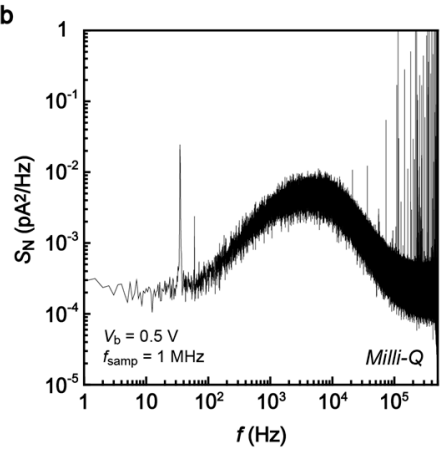

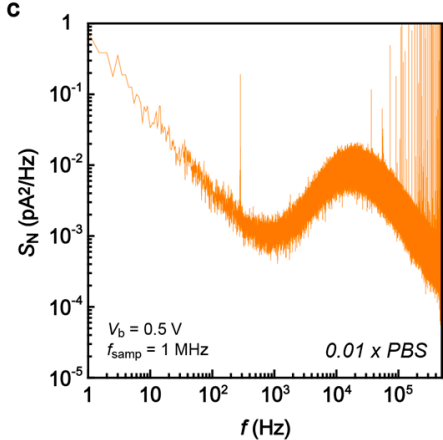

Figure 7. (a-c) Noise spectra in an air gap (a); Milli-Q (b); and $0.01 \times$ PBS (c) at $1 \mathrm{MHz}$ sampling rate with a $1 \mathrm{MHz}$ low-pass filter.

a

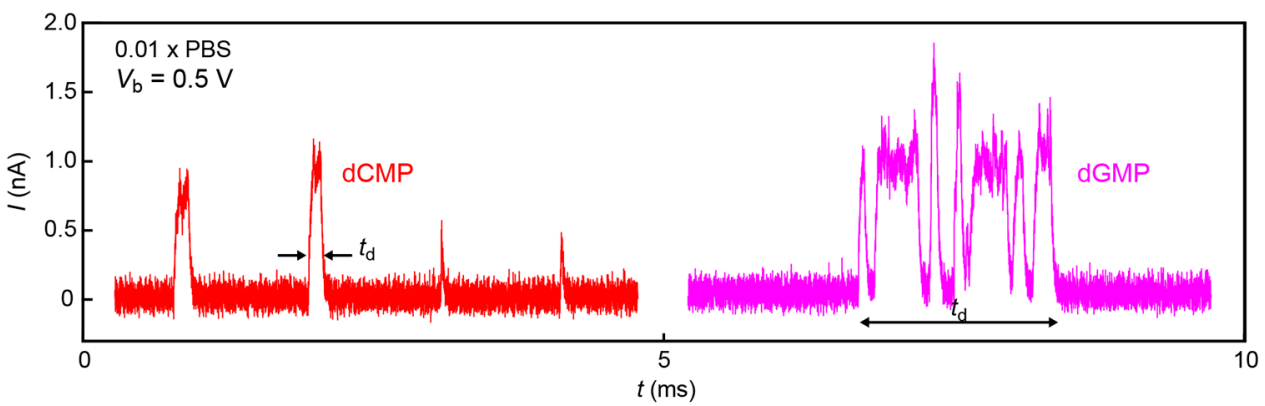

b
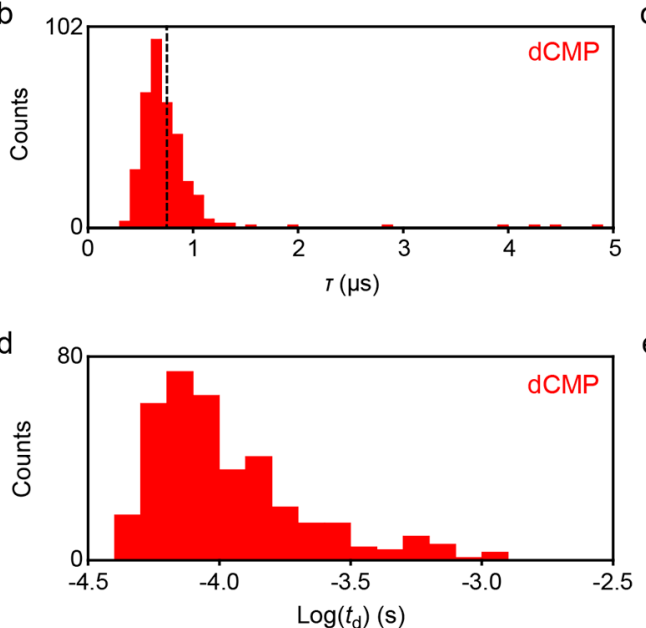
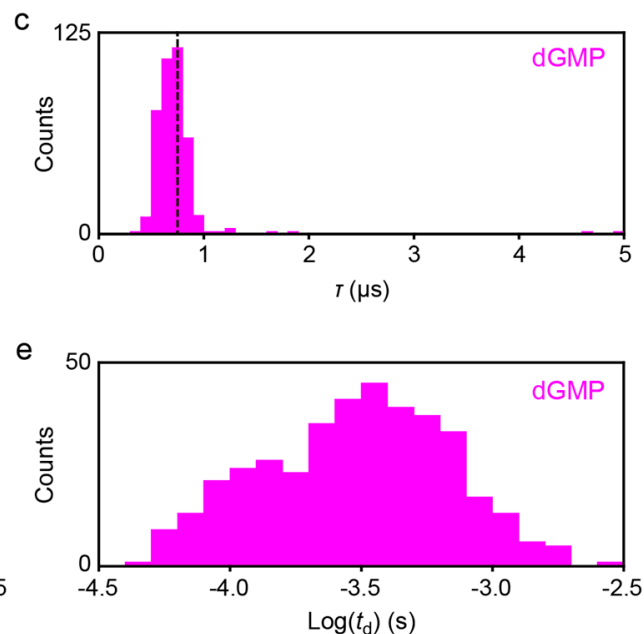

Figure 8. (a) Tunneling current spikes detected for dCMP (red) and dGMP (purple) in $0.01 \times$ PBS at $1 \mathrm{MHz}$ with a $1 \mathrm{MHz}$ low-pass filter; (b,c) Distributions of $\tau$ for dCMP (b) and dGMP (c) in $0.01 \times$ PBS. Dotted lines denote the average $\tau$ in DMSO; $(\mathbf{d}, \mathbf{e})$ Spike width $t_{\mathrm{d}}$ histograms for dCMP (d) and $\operatorname{dGMP}(\mathbf{e})$.

While no notable molecular feature was found in the $\tau$ distributions, we observed clear difference in the spike width $t_{\mathrm{d}}$. Figure $8 \mathrm{~d}$,e shows $t_{\mathrm{d}}$ histograms for dCMP and dGMP. The results reveal longer $t_{\mathrm{d}}$ of dGMP than dCMP, indicating that the former molecule tends to be trapped for a longer period in the electrode gap. This difference could not be observed conspicuously in the previous experiments due in part to the inadequate sampling rate (several $\mathrm{kHz}$ ) to correctly detect the short-lived trapping events with $t_{\mathrm{d}}$ in a range of sub-milliseconds. We hope that future works clarify the underlying mechanism of the nucleotide-dependent trap durations. 


\section{Discussion}

It is interesting to consider the source of $R C$ element relevant to the temporal resolution of the tunneling current measurements in the present setup. For the $\mathrm{SiO}_{2}$-covered $\mathrm{Au}$ nanoelectrodes, the structure can be roughly described by an equivalent circuit consisting of the resistance $R_{\mathrm{dCMP}}$ of single-molecule dCMP and the capacitance of EDL, $C_{\mathrm{EDL}}$, and the $\mathrm{SiO}_{2}$ layer, $\mathrm{C}_{\mathrm{SiO} 2}$, connected in parallel, together with the resistance of $\mathrm{Au}$ lead, $R_{\text {lead, }}$ connected in series (Figure 9). Here, $R_{\mathrm{dCMP}}=35 \mathrm{G} \Omega$ on average [4], $C_{\mathrm{SiO} 2}=W \varepsilon \varepsilon_{0} / L=7 \mathrm{pF}$ considering the surface area of $\mathrm{SiO}_{2} / \mathrm{Au}$ micro-leads of width $W$ and length $L$ of $10 \mu \mathrm{m}$ and $1 \mathrm{~mm}$, respectively (with the vacuum permittivity $\varepsilon_{0}$ and the relative permittivity of $\mathrm{SiO}_{2} \varepsilon=3.9$ ), and $C_{\mathrm{EDL}}<1 \mathrm{fF}$ at the exposed Au tip surface with an of area $100 \mathrm{~nm} \times 100 \mathrm{~nm}$. These values give $R C_{\text {gap }}$ at the electrode gap of about $0.1 \mathrm{~s}$, where $C_{\text {gap }}=\left(C_{\mathrm{SiO} 2} / 2+C_{\mathrm{EDL}} / 2\right)$ is the capacitance of the electrode gap, which is considerably longer than what is found in the tunneling current response in the present study. On the other hand, Smeets et al. [22] proposed empirically that the bandwidth of the ionic current measurements in a nanopore system is determined by the product of the membrane capacitance and the access resistance, instead of the resistance inside the pore [22,23]. Analogously, although the underlying physical mechanism is still elusive, $\tau$ in the present work may also be determined by $R_{\text {lead }}$ rather than $R_{\mathrm{dCMP}}$. In that case, tentatively assumed $R_{\text {lead }}=1 / \mathrm{G}_{0}=12.9 \mathrm{k} \Omega$ provides $R_{\text {lead }} C_{\mathrm{EDL}} / 2=7 \mu$ s with $C_{\mathrm{EDL}}=1 \mathrm{nF}$ for the non-protected MCBJs, which is in agreement with the experimental $\tau$ of $45 \mu$ s within an order of magnitude. Meanwhile, the reduced $C_{\mathrm{EDL}}$ in the $\mathrm{SiO}_{2}$-coated nanoelectrodes predicts $R_{\text {lead }} C_{\text {gap }}$ $\sim R_{\text {lead }} C_{\mathrm{SiO} 2} / 2$ of $90 \mathrm{~ns}$. This indicates that the temporal resolution is limited by a parasitic capacitance much larger than $C_{\mathrm{SiO} 2}$. Further effort to reduce the capacitance in the measurement system, for instance via analog compensation [23], may improve the temporal resolution beyond $\mathrm{MHz}$.

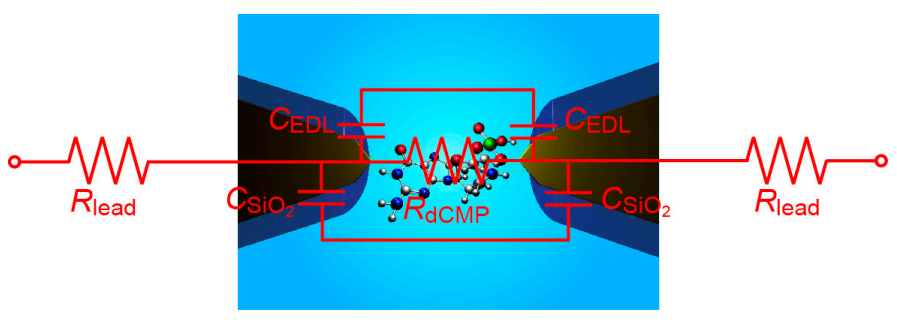

Figure 9. Rough circuit model of the $\mathrm{SiO}_{2} / \mathrm{Au}$ nanoelectrodes consisting of the single-molecule resistance $\left(R_{\mathrm{dCMP}}\right)$, the capacitance of the electric double layers $\left(C_{\mathrm{EDL}}\right)$ and $\mathrm{SiO}_{2}$ layers $\left(C_{\mathrm{SiO} 2}\right)$, and the Au lead resistance $\left(R_{\text {lead }}\right)$.

\section{Conclusions}

We performed single-nucleotide detections by fast tunneling current measurements at $1 \mathrm{MHz}$ sampling rates using $\mathrm{SiO}_{2}$-protected nanoelectrodes. We observed rapid fluctuations of the current within individual spike signals, signifying dynamic molecular conformation changes in the electrode gap. Meanwhile, blunt features were found in the single-molecule traces, which indicated signal retardation by $R C$ effects limiting the temporal resolution to around $7 \mu \mathrm{s}$. Further efforts to reduce the capacitance may enable fine tracking of tunneling current changes in an aqueous solution for studying single-molecule dynamics at high spatiotemporal resolutions, thus delivering one of the essential technologies to realize sequencing by quantum mechanics.

Acknowledgments: This work was supported by the Japan Society for the Promotion of Science (JSPS) KAKENHI Grant No. 26220603 and "Nanotechnology Platform Project (Nanotechnology Open Facilities in Osaka University)" of Ministry of Education, Culture, Sports, Science and Technology, Japan (No. F-12-OS-0016).

Author Contributions: Makusu Tsutsui and Masateru Taniguchi conceived and designed the experiments; Takanori Morikawa, Sachie Tanimoto, and Kazumichi Yokota performed the experiments; Takanori Morikawa, Makusu Tsutsui and Kazumichi Yokota analyzed the data; Makusu Tsutsui and Masateru Taniguchi co-wrote the paper. 
Conflicts of Interest: The authors declare no conflict of interest.

\section{References}

1. Di Ventra, M.; Taniguchi, M. Decoding DNA, RNA and peptides with quantum tunneling. Nat. Nanotechnol. 2016, 11, 117-126. [CrossRef] [PubMed]

2. Lindsay, S. The promises and challenges of solid-state sequencing. Nat. Nanotechnol. 2016, 11, $109-111$. [CrossRef] [PubMed]

3. Yokota, K.; Tsutsui, M.; Taniguchi, M. Electrode-embedded nanopores for label-free single-molecule sequencing by electric currents. RSC Adv. 2014, 4, 15886-15889. [CrossRef]

4. Tsutsui, M.; Taniguchi, M.; Yokota, K.; Kawai, T. Identifying single nucleotides by tunneling current. Nat. Nanotechnol. 2010, 5, 286-290. [CrossRef] [PubMed]

5. Huang, S.; He, J.; Chang, S.; Zhang, P.; Liang, F.; Li, S.; Tuchband, M.; Fuhrmann, A.; Ros, R.; Lindsay, S. Identifying single bases in a DNA oligomer with electron tunneling. Nat. Nanotechnol. 2010, 5, 868-873. [CrossRef] [PubMed]

6. Pang, P.; Ashcroft, B.A.; Song, W.; Zhang, P.; Biswas, S.; Qing, Q.; Yang, J.; Nemanich, R.J.; Bai, J.; Smith, J.T.; et al. Fixed-gap tunnel junction for reading DNA nucleotides. ACS Nano 2014, 8, 11994-12003. [CrossRef] [PubMed]

7. Ohshiro, T.; Tsutsui, M.; Yokota, K.; Furuhashi, M.; Taniguchi, M.; Kawai, T. Detection of post-translational modifications in single peptides using electron tunnelling currents. Nat. Nanotechnol. 2014, 9, 835-840. [CrossRef] [PubMed]

8. Zhao, Y.; Ashcroft, B.; Zhang, P.; Liu, H.; Sen, S.; Song, W.; Im, J.; Gyarfas, B.; Manna, S.; Biswas, S.; et al. Single-molecule spectroscopy of amino acids and peptides by recognition tunnelling. Nat. Nanotechnol. 2013, 9, 466-473. [CrossRef] [PubMed]

9. Ohshiro, T.; Matsubara, K.; Tsutsui, M.; Furuhashi, M.; Taniguchi, M.; Kawai, T. Single-molecule electrical random resequencing of DNA and RNA. Sci. Rep. 2012, 2, 501. [CrossRef] [PubMed]

10. Chang, S.; Huang, S.; He, J.; Liang, F.; Zhang, P.; Li, S.; Chen, X.; Sankey, O.; Lindsay, S. Electronic signatures of all DNA nucleosides in a tunneling gap. Nano Lett. 2010, 10, 1070-1075. [CrossRef] [PubMed]

11. Tsutsui, M.; Rahong, S.; Iizumi, Y.; Okazaki, T.; Taniguchi, M.; Kawai, T. Single-molecule sensing electrode embedded in-plane nanopore. Sci. Rep. 2011, 1, 46. [CrossRef] [PubMed]

12. Ivanov, A.P.; Instuli, E.; McGilvery, C.M.; Baldwin, G.; McComb, D.W.; Albrecht, T.; Edel, J.B. DNA tunneling detector embedded in a nanopore. Nano Lett. 2011, 11, 279-285. [CrossRef] [PubMed]

13. Lagerqvist, J.; Zwolak, M.; Di Ventra, M. Fast DNA sequencing via transverse electronic transport. Nano Lett. 2006, 6, 779-782. [CrossRef] [PubMed]

14. Ivanov, A.P.; Freedman, K.J.; Kim, M.J.; Albrecht, T.; Edel, J.B. High precision fabrication and positioning of nanoelectrodes in a nanopore. ACS Nano 2014, 8, 1940-1948. [CrossRef] [PubMed]

15. Fanget, A.; Traversi, F.; Khlybov, S.; Granjon, P.; Magrez, A.; Forró, L.; Radenovic, A. Nanopore integrated nanogaps for DNA detection. Nano Lett. 2014, 14, 244-249. [CrossRef] [PubMed]

16. Arima, A.; Tsutsui, M.; Morikawa, T.; Yokota, K.; Taniguchi, M. Fabrications of insulator-protected nanometer-sized electrode gaps. J. Appl. Phys. 2014, 115, 114310. [CrossRef]

17. Muthusubramanian, N.; Galan, E.; Maity, C.; Eelkema, R.; Grozema, F.C.; van der Zant, H.S.J. Insulator-protected mechanically controlled break junctions for measuring single-molecule conductance in aqueous environments. Appl. Phys. Lett. 2016, 109, 013102. [CrossRef]

18. Tanimoto, S.; Tsutsui, M.; Yokota, K.; Taniguchi, M. Dipole effects on the formation of molecular junctions. Nanoscale Horiz. 2016, 1, 399-406. [CrossRef]

19. Morikawa, T.; Yokota, K.; Tsutsui, M.; Taniguchi, M. Fast and low-noise tunnelling current measurements for single-molecule detections in electrolyte solution using insulator-protected nanoelectrodes. Nanoscale 2017, 12, 4076-4081. [CrossRef] [PubMed]

20. Rosenstein, J.K.; Wanunu, M.; Merchant, C.A.; Drndic, M.; Shepard, K.L. Integrated nanopore sensing platform with sub-microsecond temporal resolution. Nat. Methods 2012, 9, 487-494. [CrossRef] [PubMed]

21. Freed, B.K.; Biesecker, J.; Middleton, W.J. Spectral polarity index: A new method for determining the relative polarity of solvents. J. Flour. Chem. 1990, 48, 63-75. [CrossRef] 
22. Smeets, R.M.M.; Keyser, U.F.; Dekker, N.H.; Dekker, C. Noise in solid-state nanopores. Proc. Natl. Acad. Sci. USA 2008, 105, 417-421. [CrossRef] [PubMed]

23. Dimitrov, V.; Mirsaidov, U.; Wang, D.; Sorsch, T.; Mansfield, W.; Miner, J.; Klemens, F.; Cirelli, R.; Yemenicioglu, S.; Timp, G. Nanopores in solid-state membranes engineered for single molecule detection. Nanotechnology 2010, 21, 065502. [CrossRef] [PubMed] article distributed under the terms and conditions of the Creative Commons Attribution (CC BY) license (http:/ / creativecommons.org/licenses/by/4.0/). 